\title{
Chemokine (C-X-C Motif) Receptor 4 Regulates Lung Endothelial Barrier Permeability During Resuscitation from Hemorrhagic Shock
}

\author{
F. S. BABU ${ }^{1}$, H. M. LAPORTE ${ }^{1}$, S. P. NASSOIY ${ }^{1}$, M. MAJETSCHAK ${ }^{2,3}$ \\ ${ }^{1}$ Department of Surgery, Burn and Shock Trauma Research Institute, Loyola University Chicago, \\ Stritch School of Medicine, Maywood, IL, USA, ${ }^{2}$ Department of Surgery, University of South \\ Florida Morsani College of Medicine, Tampa, FL, USA, ${ }^{3}$ Department of Molecular Pharmacology \\ and Physiology, University of South Florida Morsani College of Medicine, Tampa, FL, USA
}

Received December 18, 2018

Accepted April 4, 2019

Epub Ahead of Print June 6, 2019

\section{Summary}

Chemokine (C-X-C motif) receptor 4 (CXCR4) agonists have been shown to protect lung endothelial barrier function in vitro. In vivo effects of CXCR4 modulation on lung endothelial permeability are unknown. Here we tested the effects of the CXCR4 agonist ubiquitin and the antagonist AMD3100 on lung vascular permeability and cytokine concentrations in a rat hemorrhage model. Animals were hemorrhaged (mean arterial blood pressure $30 \mathrm{mmHg}$ for $30 \mathrm{~min}$ ), treated with vehicle, ubiquitin (0.7 and $3.5 \mu \mathrm{mol} / \mathrm{kg})$ or AMD3100 $(3.5 \mu \mathrm{mol} / \mathrm{kg})$, and resuscitated with crystalloids. Evans blue extravasation was employed to quantify lung vascular permeability. Ubiquitin dose-dependently reduced Evans blue extravasation into the lung. AMD3100 increased Evans blue extravasation. With AMD3100, TNFa levels in lung homogenates were increased; while TNFa levels were lower with ubiquitin, these differences did not reach statistical significance. Our findings suggest that CXCR4 regulates lung vascular permeability and further point towards CXCR4 as a drug target to confer lung protection during resuscitation from traumatichemorrhagic shock.

\section{Key words}

CXCR4 • Ubiquitin • AMD3100 • Endothelial permeability • Lung

\section{Corresponding author}

M. Majetschak, Department of Surgery, Morsani College of Medicine, University of South Florida, 12901 Bruce B Downs Blvd., MDC 3127, Tampa, Florida 33612, USA. E-mail: majetschak@health.usf.edu
Development of acute respiratory distress syndrome (ARDS) remains a major contributor to morbidity and mortality in critically ill trauma patients (Blank and Napolitano 2011, Ranieri et al. 2012, Dushianthan et al. 2011, Gonzales et al. 2015). While impairment of pulmonary endothelial barrier function in response to local and systemic coagulation and inflammation is considered the hallmark in the development of ARDS, the underlying pathophysiology is not well defined. Therapeutic strategies to protect lung endothelial barrier function during the early inflammatory response to traumatic-hemorrhagic shock and to reduce the risk of ARDS development are not available. Such strategies, however, would be desirable as they have the potential to improve survival and reduce morbidity in trauma patients. Several lines of pre-clinical evidence suggest that activation of chemokine (C-X-C motif) receptor 4 (CXCR4) confers lung protection in various models of lung injury and ARDS (Majetschak et al. 2004, Garcia-Covarrubias et al. 2008, Guo et al. 2016, Baker et al. 2012, Nassoiy et al. 2018). Furthermore, the natural CXCR4 agonists (C-X-C motif) chemokine ligand 12 (CXCL12) and ubiquitin, and CTCE-0214, a synthetic CXCL12 analogue, have previously been shown to attenuate thrombin-induced impairment of lung endothelial barrier function in vitro (Kobayashi et al. 2014, Guo et al. 2016, Cheng et al. 2017). Whether pharmacological modulation of CXCR4 influences lung endothelial barrier function in vivo, however, is unknown. 
Thus, we tested whether pharmacological activation or blockade of CXCR4 affects lung endothelial barrier function during the inflammatory response to hemorrhagic shock and resuscitation in a rat model. We utilized Evans blue extravasation to quantify in vivo lung vascular permeability as the primary study endpoint. All procedures were performed according to National Institutes of Health Guidelines for Use of Laboratory Animals and approved by the Institutional Animal Care and Use Committee. Male Sprague Dawley rats (300-350 g) were purchased from Harlan (Indianapolis, IN, USA). Anesthetized (isoflurane inhalation) animals were oro-tracheally intubated with a 16-gauge EXEL disposable safelet angiocatheter (EXELINT International, Los Angeles, CA, USA) and mechanically ventilated with a SomnoSuite small animal anesthesia system (Kent Scientific Corporation, Torrington, CT, USA). Animals were ventilated with an initial positive end expiratory pressure (PEEP) of $2 \mathrm{mmHg}$, a fraction of inspired oxygen $\left(\mathrm{FiO}_{2}\right)$ of 1.0 and anesthetized with $2.5 \%$ isoflurane. Tidal volumes were titrated to maintain normal $\mathrm{PaCO}_{2}(35-45 \mathrm{mmHg})$. The femoral artery was then cannulated with a 24-gauge BD angiocath shielded IV catheter (Becton, Dickinson and Company, Franklin Lakes, NJ, USA) to allow for monitoring of arterial blood pressure and blood withdrawal. The femoral vein was cannulated with 1.5-french tubing for fluid and drug administration. After instrumentation and recording of baseline parameters, animals were hemorrhaged to mean arterial blood pressure (MAP) of $30 \mathrm{mmHg}$ for $30 \mathrm{~min}$. In series 1 , vehicle ( $1.1 \mathrm{ml}$ normal saline (NS), $\mathrm{n}=5)$, $0.7(\mathrm{n}=5)$ or $3.5(\mathrm{n}=5) \mu \mathrm{mol} / \mathrm{kg}$ ubiquitin in $1.1 \mathrm{ml} \mathrm{NS}$ were injected intravenously at $\mathrm{t}=30 \mathrm{~min}$. In series 2 , $1.1 \mathrm{ml}$ vehicle $(\mathrm{n}=6)$ or $3.5 \mu \mathrm{mol} / \mathrm{kg}$ AMD3100 $(\mathrm{n}=5)$ in $1.1 \mathrm{ml} \mathrm{NS}$ were injected intravenously at $\mathrm{t}=30 \mathrm{~min}$. In both series, drugs were administered randomized and blinded. Immediately after drug administration, animals were resuscitated with 1.5-times of their blood volume of warmed NS from $\mathrm{t}=30 \mathrm{~min}$ to $\mathrm{t}=55 \mathrm{~min}$. At $\mathrm{t}=90 \mathrm{~min}$, $0.5 \%$ Evans blue solution in phosphate buffered saline
A

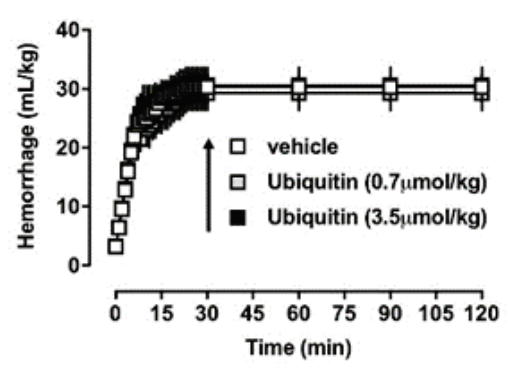

D

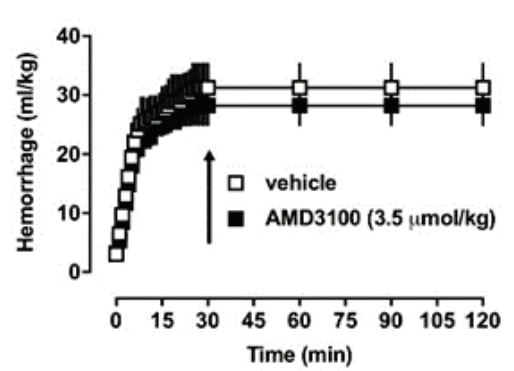

B

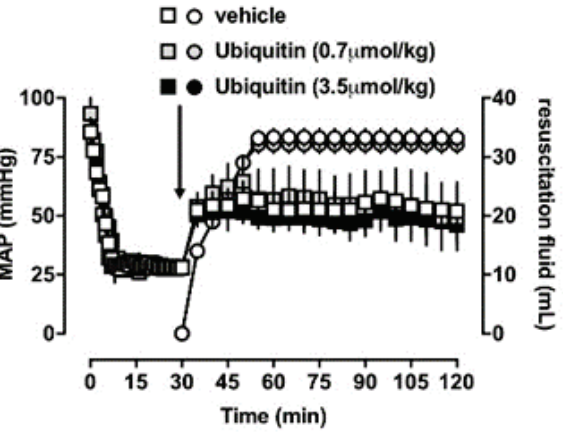

$\mathbf{E}$

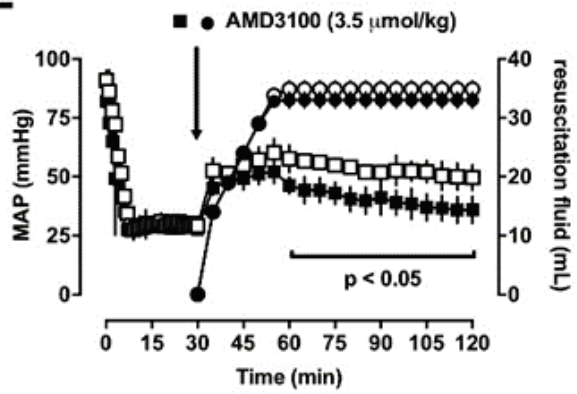

C

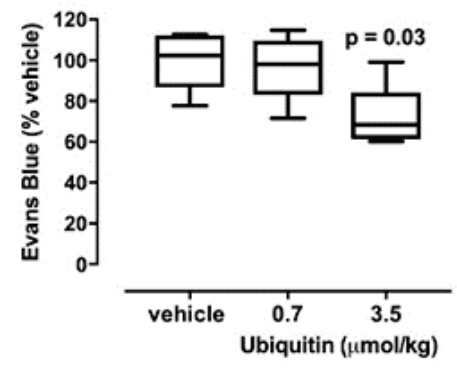

$\mathbf{F}$

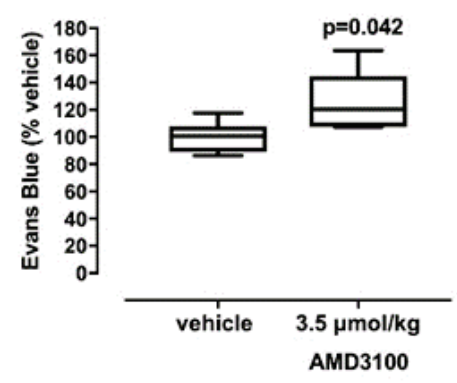

Fig. 1. Effects of pharmacological CXCR4 modulation on lung vascular permeability after hemorrhagic shock and fluid resuscitation. Animals were hemorrhaged to a mean arterial blood pressure (MAP) of $30 \mathrm{mmHg}$, followed by standardized fluid resuscitation. Arrows show time points of drug administration. A/B: Open symbols: vehicle. Grey symbols: Ubiquitin $0.7 \mu \mathrm{mol} / \mathrm{kg}$. Black symbols: Ubiquitin $3.5 \mu \mathrm{mol} / \mathrm{kg}$. Data are mean \pm standard deviation. A. Hemorrhage volume $(\mathrm{ml} / \mathrm{kg})$. B. Squares: MAP (mm Hg). Circles: Resuscitation fluid ( $\mathrm{ml}$ ). C. Evans blue concentrations in lung homogenates from animals in A/B. Data are expressed as \% of vehicle treated animals. Boxes extend from the $25^{\text {th }}-75^{\text {th }}$ percentiles; horizontal lines show the median; whiskers indicate minimum/maximum. Statistically significant differences are indicated. D/E: Open symbols: vehicle. Black symbols: AMD3100 $3.5 \mu \mathrm{mol} / \mathrm{kg}$. Data are mean \pm standard deviation. D. Hemorrhage volume $(\mathrm{ml} / \mathrm{kg})$. E. Squares: MAP $(\mathrm{mmHg})$. Circles: Resuscitation fluid $(\mathrm{ml})$. Significant differences in MAP between groups are indicated. F. Evans blue concentrations in lung homogenates from animals in D/E. Data are expressed as \% of vehicle treated animals. Boxes extend from the $25^{\text {th }}-75^{\text {th }}$ percentiles; horizontal lines show the median; whiskers indicate minimum/maximum. Statistically significant differences are indicated. 
(25 mg/kg) were administered intravenously. At $\mathrm{t}=120 \mathrm{~min}$, a bilateral thoracotomy was performed, and the rats were exsanguinated. The right ventricle was isolated from the systemic circulation and the pulmonary artery was cannulated and flushed with $20 \mathrm{ml}$ of phosphate buffered saline until clear effluent drained from the femoral arterial catheter. Bilateral bronchioalveolar lavage (BAL) was performed and lung specimens were harvested. Tissue samples were weighed, homogenized in formamide (1.5 weight/volume) and centrifuged (16000 g, $\left.15 \mathrm{~min}, 4{ }^{\circ} \mathrm{C}\right)$; the supernatant was collected, and absorbance was measured at $610 \mathrm{~nm}$ in a microplate reader. Cytokine concentrations (interleukin (IL)-6, IL-10, tumor necrosis factor (TNF $\alpha)$ in tissue homogenates were measured using the DuoSet ELISA Development kit (R\&D Systems), as recommended by the manufacturer. Protein concentrations were measured with the Bio-Rad RC DC Protein Assay kit. Arterial blood gases and routine laboratory parameters were analyzed using the Element point of care veterinary blood gas, electrolyte and critical care analyzer (Cuattro Veterinary USA, Loveland, CO, USA). Data were analyzed with 1-way or 2-way ANOVA with Bonferroni's multiple comparisons test or Student's t-test, as appropriate. A 2-tailed $\mathrm{p}<0.05$ was considered significant. All data analyses were performed with the GraphPad Prism 7 program. Data are described as mean \pm standard deviation.

There were no differences in any physiological parameters among groups at baseline. Hemorrhage volumes (Fig. 1A), MAP and volumes of resuscitation fluids (Fig. 1B) were comparable among animals in series 1 . With $0.7 \mu \mathrm{mol} / \mathrm{kg}$ ubiquitin, Evans blue concentrations in lung homogenates were $97 \pm 16 \%$ ( $p>0.05$ vs. vehicle), and with $3.5 \mu \mathrm{mol} / \mathrm{kg}$ ubiquitin $72 \pm 16 \%$ of vehicle treated animals $(100 \pm 15 \%, \mathrm{p}<0.05)$ (Fig. 1C). These observations are in line with the previously described effects of ubiquitin on tissue wet/dry weight ratios in a porcine polytrauma model with direct lung injury and in a rat unilateral lung ischemiareperfusion injury model (Baker et al. 2012, GarciaCovarrubias et al. 2008). Furthermore, our observation that ubiquitin treatment reduces Evans blue extravasation into the lung compares well with the protective effects of the CXCR4 agonists ubiquitin and CXCL12 on thrombininduced impairment of lung endothelial barrier function in vitro (Cheng et al. 2017). To assess the role of the endogenous CXCR4 agonists in the regulation of lung endothelial barrier function during resuscitation from

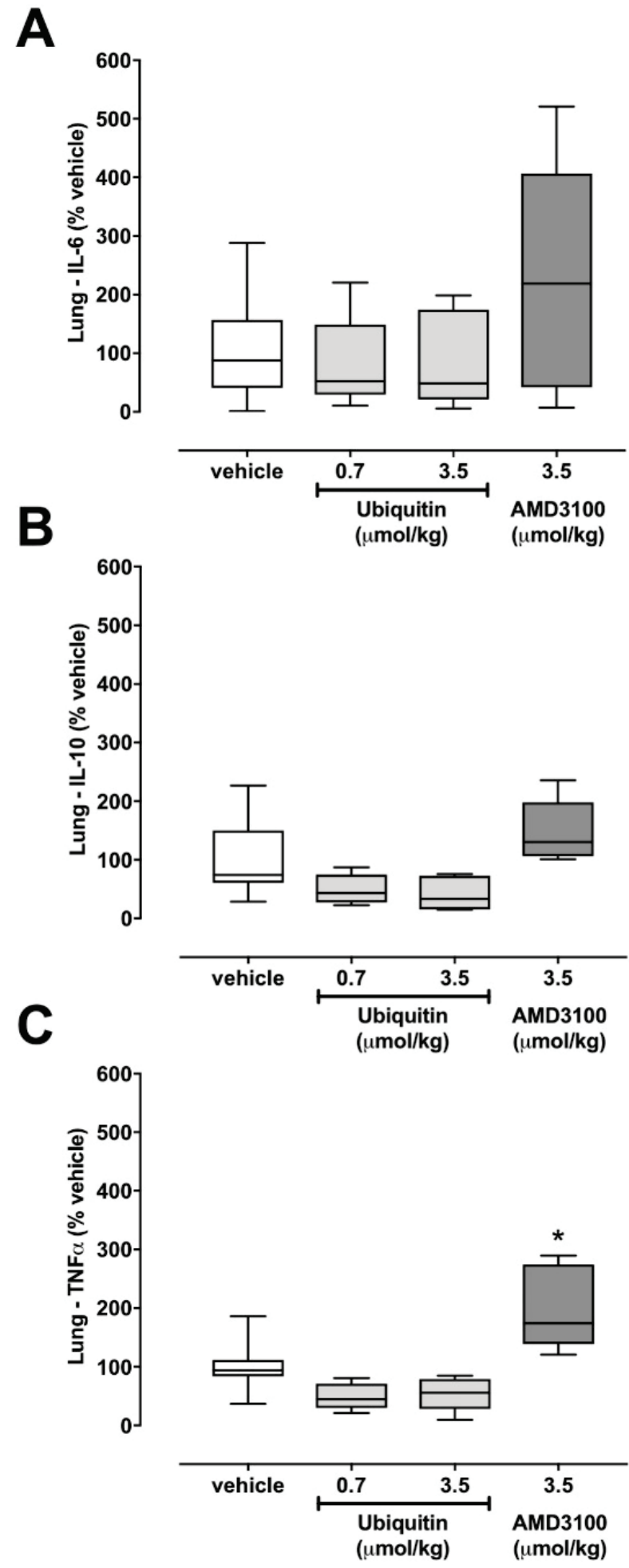

Fig. 2. Cytokine concentrations in lung homogenates. Cytokine concentrations per $\mathrm{mg}$ total protein are expressed as $\%$ of vehicle treated animals. Boxes extend from the $25^{\text {th }}-75^{\text {th }}$ percentiles; horizontal lines show the median; whiskers indicate minimum/maximum. Statistically significant differences are indicated. $* \mathrm{p}<0.05$ vs. vehicle and ubiquitin groups. A. IL-6. B. IL-10. C. TNFa.

hemorrhagic shock, we then performed series 2 and compared the effects of the CXCR4 antagonist 
AMD3100 with vehicle treatment. AMD3100 was administered in a dose equimolar to the dose of ubiquitin that reduced Evans blue extravasation in series 1. While hemorrhage volumes were comparable between groups (Fig. 1D) and volumes of resuscitation fluid volumes were indistinguishable between the vehicle and the AMD3100 groups, MAP was significantly lower with AMD3100 treatment from $t=60-120$ min (Fig. 1E). This observation is consistent with the effects of AMD3100 on blood pressures and fluid requirements that have been described previously in other rat and pig traumahemorrhage models (Bach et al. 2016, Bach et al. 2014).

As compared with vehicle-treated animals, Evans blue concentrations in lung homogenates were significantly increased with AMD3100 treatment $(100 \pm 11 \%$ vs. $125 \pm 23 \%, \mathrm{p}<0.05)$. Our findings that ubiquitin and AMD3100 have diametrically opposing effects on Evans blue extravasation into the lung compare well with the observations that AMD3100 treatment exerts effects opposite to the effects of ubiquitin in trauma models and neutralizes the effects of ubiquitin when co-administered in equimolar concentrations (Bach et al. 2012, Bach et al. 2016, Bach et al. 2014). The previous finding that AMD3100 does not affect human pulmonary endothelial cell monolayer barrier function in vitro is not contradictive because these experiments were performed in the absence of endogenous CXCR4 agonists (Cheng et al. 2017). Thus, the results of the present study suggest that endogenous CXCR4 agonists have lung protective functions and that treatment with such agonists further improves lung endothelial barrier function in vivo.

In both series, blood gases, plasma electrolyte and lactate concentrations and protein concentrations in BAL fluids were comparable among groups ( $p>0.05$ for all parameters, not shown). Our findings that blood gases were within the normal range in all animals and that BAL protein concentrations were low $(<0.3 \mathrm{mg} / \mathrm{ml}$ in all groups) were anticipated because of the short observation period in the present study, which does not permit the development of ARDS or significant protein leakage into the alveolar space.

To assess whether pharmacological CXCR4 modulation also affects inflammation markers in the lung parenchyma, we measured TNF $\alpha$, IL-6 and IL-10 as prototypical pro- and anti-inflammatory inflammation markers in extracts from lungs after removal of blood from the lung vasculature. While there were no statistically significant differences among groups for IL-6 and IL-10 concentrations per mg total protein in lung homogenates
(Fig. 2A/B), TNF $\alpha$ concentrations per mg total protein were significantly increased with AMD3100 treatment, as compared with vehicle and ubiquitin treatment (Fig. 2C). While TNF $\alpha$ levels were lower with ubiquitin treatment, these differences did not reach statistical significance in post-hoc testing. Nevertheless, it appears worthwhile mentioning that the degree by which ubiquitin treatment reduced Evans blue extravasation and $\mathrm{TNF} \alpha$ levels in lung homogenates was comparable with the degree by which AMD3100 exerted opposite effects. Furthermore, the findings from the present study confirm previous findings in other trauma models, which indicated that CXCR4 activation reduces inflammation markers that are generally considered as pro-inflammatory (Garcia-Covarrubias et al. 2008, Bach et al. 2012).

In conclusion, in the present study, we provide initial evidence that CXCR4 activation reduces, whereas CXCR4 blockade enhances in vivo lung vascular permeability during resuscitation from hemorrhagic shock. While the blood pressure lowering effects of AMD3100 are consistent with previous observations in other hemorrhage and resuscitation models, it remains to be determined whether these effects are caused by reduced intravascular volumes due to impaired endothelial barrier function with subsequent third spacing of fluids, by direct effects on vascular smooth muscle function or by a combination of both (Bach et al. 2014). In vivo experiments to delineate the underlying mechanisms, however, are beyond the scope of the present study. Nevertheless, our findings demonstrate that in vitro effects of CXCR4 modulation on lung endothelial barrier function can be translated in vivo (Cheng et al. 2017) and further suggest CXCR4 as a drug target to provide lung protection during the inflammatory response to trauma, hemorrhage and fluid resuscitation (Nassoiy et al. 2018).

\section{Conflict of Interest}

There is no conflict of interest.

\section{Acknowledgements}

This research has been presented in part at the $41^{\text {st }}$ Annual Conference on Shock, Scottsdale, Arizona, USA, June 2018. This work was supported by the Office of the Assistant Secretary of Defense for Health Affairs through the Peer Reviewed Medical Research Program under Award No. W81XWH-15-1-0262 and by the National Institute of General Medical Sciences (Awards R01GM107495 and T32GM008750). The content is solely the responsibility of the authors. 


\section{References}

BACH HH, SAINI V, BAKER TA, TRIPATHI A, GAMELLI RL, MAJETSCHAK M: Initial assessment of the role of CXC chemokine receptor 4 after polytrauma. Mol Med 18: 1056-1066, 2012.

BACH HH, WONG YM, LAPORTE HM, GAMELLI RL, MAJETSCHAK M: Pharmacological targeting of chemokine (C-X-C motif) receptor 4 in porcine polytrauma and hemorrhage models. J Trauma Acute Care Surg 80: 102-110, 2016.

BACH HH, WONG YM, TRIPATHI A, NEVINS AM, GAMELLI RL, VOLKMAN BF, BYRON KL, MAJETSCHAK M: Chemokine (C-X-C motif) receptor 4 and atypical chemokine receptor 3 regulate vascular alpha(1)-adrenergic receptor function. Mol Med 20: 435-447, 2014.

BAKER TA, ROMERO J, BACH HH, STROM JA, GAMELLI RL, MAJETSCHAK M: Effects of exogenous ubiquitin in a polytrauma model with blunt chest trauma. Crit Care Med 40: 2376-2384, 2012.

BLANK R, NAPOLITANO LM: Epidemiology of ARDS and ALI. Crit Care Clin 27: 439-458, 2011.

CHENG YH, EBY JM, LAPORTE HM, VOLKMAN BF, MAJETSCHAK M: Effects of cognate, non-cognate and synthetic CXCR4 and ACKR3 ligands on human lung endothelial cell barrier function. PLoS One 12: e0187949, 2017.

DUSHIANTHAN A, GROCOTT MP, POSTLE AD, CUSACK R: Acute respiratory distress syndrome and acute lung injury. Postgraduate medical journal 87: 612-622, 2011.

GARCIA-COVARRUBIAS L, MANNING EW, 3RD, SORELL LT, PHAM SM, MAJETSCHAK M: Ubiquitin enhances the Th2 cytokine response and attenuates ischemia-reperfusion injury in the lung. Crit Care Med 36: 979-982, 2008.

GONZALES JN, LUCAS R, VERIN AD: The acute respiratory distress syndrome: mechanisms and perspective therapeutic approaches. Austin J Vasc Med 2: 1009, 2015.

GUO C, GOODWIN AJ, BUIE JN, COOK JA, HALUSHKA PV, ARGRAVES K, ZINGARELLI B, ZHANG XK, WANG L, FAN H: A stromal cell-derived factor 1 alpha analogue improves endothelial cell function in lipopolysaccharide-induced acute respiratory distress syndrome. Mol Med 22: 115-123, 2016.

KOBAYASHI K, SATO K, KIDA T, OMORI K, HORI M, OZAKI H, MURATA T: Stromal cell-derived factor1alpha/C-X-C chemokine receptor type 4 axis promotes endothelial cell barrier integrity via phosphoinositide 3-kinase and Rac1 activation. Arterioscler Thromb Vasc Biol 34: 1716-1722, 2014.

MAJETSCHAK M, COHN SM, NELSON JA, BURTON EH, OBERTACKE U, PROCTOR KG: Effects of exogenous ubiquitin in lethal endotoxemia. Surgery 135: 536-543, 2004.

NASSOIY SP, BABU FS, LAPORTE HM, MAJETSCHAK M: Pharmacological modulation of C-X-C motif chemokine receptor 4 influences development of acute respiratory distress syndrome after lung ischaemiareperfusion injury. Clin Exp Pharmacol Physiol 45: 16-26, 2018.

RANIERI VM, RUBENFELD GD, THOMPSON BT, FERGUSON ND, CALDWELL E, FAN E, CAMPOROTA L, SLUTSKY AS: Acute respiratory distress syndrome: The Berlin Definition. JAMA 307: 2526-2533, 2012. 